Bueno de Santiago, Alejandra.

Doctoranda en el programa de doctorado Arte e investigación. Universidad Politécnica de Valencia, Facultad de Bellas Artes de San Carlos.

\title{
Experiencias reales a través de conocimientos virtuales, un nuevo estado de derecho a través del arte y la tecnología. La obsolescencia de lo virtual.
}

\author{
TIPO DE TRABAJO \\ Comunicación virtual. \\ PALABRAS CLAVE \\ videoarte, interactivo, cuerpo, virtual.
}

KEY WORDS

Experiences, videoart, interactive, body, virtual.

RESUMEN

El cuerpo evoluciona de forma natural mientras que el entorno se convierte cada vez más en objetos técnicos conectados a la red que pretenden ser controlados por nuestros cuerpos reales, se trata de un viaje hacia lo virtual para representar lo real manipulado por los avances tecnológicos. Pero estos cuerpos, carecen de los medios necesarios para relacionarse con este nuevo entorno. En este articulo se habla de las relaciones del cuerpo y las personas con los entornos virtuales y lo que esto genera, enfatizando el posible accidente que se puede generar con esta innovación y desarrollo técnico que sobrepasa al ser humano y lo eliminan, obviando lo más básico del ser, que son las relaciones humanas y la palabra. El objetivo es reflexionar y cuestionar este nuevo modo de vida que oscila entre el mundo virtual y el real a través del arte y sus manifestaciones dentro del campo del vídeo y la instalación.

\section{ABSTRACT}

The body naturally is developed, while the environment becomes increasingly in technical objects connected to the network that are intended to be controlled by our real bodies, it is a journey into the virtual world to represent the real world manipulated by technological advances. But these bodies lack the necessary means, to interact with this new environment. In this article we talk about the relationship of the body and people with virtual environments and what it generates, emphasizing the possible accident that can be generated with this innovation and technical development that surpasses the human being and remove it, ignoring the basics the human being, which are the human relationships and the word. The aim is to talk and questioning this new way of life that oscillates between the virtual world and the real through art and its manifestations in the field of video and installation.

Podemos entender que nuestra realidad actual viene dada por la información y el conocimiento que se genera de la observación de la realidad a través de los medios tecnológicos, es decir nos llega una versión de la realidad convertida en virtual que es interpretada por nuestro cerebro para reconvertirla en nuestra propia realidad. Dicho así parece un trabalenguas que no tiene fin, pero se quiere poner en cuestionamiento la veracidad y durabilidad de las experiencias multimedia en general, y analizar las que son llevadas a cabo por en instalaciones interactivas dentro del arte, por medio de la acción, hay una toma de conciencia e identificación dentro del sistema narrativo de la propia obra de vídeo interactiva, donde se consigue una experiencia real que integra componentes virtuales y reales en un mismo espacio y tiempo. 


\section{CONTENIDO}

Comprendemos que este mundo cada vez se ajusta más a las necesidades del ser humano por la propia acción y manipulación de este a su antojo y semejanza. Es el juego de jugar a ser Dios, primero imitamos a la naturaleza en si misma, los árboles, las plantas... ahora hemos comprendido que el objetivo máximo es imitar al ser humano y recrear sus situaciones y experiencias en espacios virtuales hechos a la medida de cada uno para complacer los deseos de los individuos conectados a la vida mediante el uso de las tecnologías. Somos capaces de diseñar elementos de apoyo a nuestras vidas que nos conozcan tan bien que no sea necesario su activación, sino que tras el estudio del comportamiento del ser humano generamos máquinas que se auto-enseñan y se programan en función de lo que hacemos o decimos. Un ejemplo básico de ello son los sistemas de detección de rostro capaces de identificar cuando una persona sonríe o no y modificar sus variables en función de este estado. No nos hace falta ni hablar ni pulsar un botón para controlar el sistema. Un simple gesto es suficiente para interactuar mediante un entorno virtual, con lo real, y generar experiencias híbridas que quedan grabadas en nuestra retina como experiencias que se generar en un solo plano, el real. Un ejemplo más claro aún es el que nos muestra el libro Big Data ${ }^{1}$, que se tiene que ver con todas las acciones que se realizan en internet, como búsquedas, clics de "me gusta", publicaciones de twiter, pues bien, todas estas acciones quedan registradas y analizadas por empresas y por instituciones, esto son los datos masivos, Big Data. Esto conlleva una nueva forma de pensar basada en la correlación, concepto que nos permite saber, que cuando se dan una serie de circunstancias, también se van a dar otras por lo que podemos capturar patrones de comportamiento para identificar personas que pertenezcan a determinado sector social, político, artístico o a determinado rango de edad, etc... Como vemos hemos sido capaces de automatizar nuestro entorno para que este reaccione a nuestros deseos. El arte por su parte tiene el papel de ocuparse más de contar y cuestionar que de medir, pues "sucede que cuando está casi todo medido, faltan aún muchas cosas que contar como contar lo que falta" ${ }^{2}$. Tras haber estudiado obras de artistas que exploran el campo correlacional existente entre la pieza y el usuario nos disponemos a analizar esta correlación y sus consecuencias en el mundo real de las relaciones sociales y en la observación y asimilación de los acontecimientos. Podemos suponer que estas interacciones entre ambos mundos pueden llegar a ocasionar confusión en aquellas personas que han nacido con el uso de las tecnologías integrado en sus vidas de manera racional y lógica, como algo simplemente normal y real. Por ejemplo queremos ejemplificar esto con una situación real que hemos podido ver en nuestras casas con los más pequeños. Una conversación por skype en la que la persona se materializa en una pantalla, su imagen real es imitada por lo virtual y representada por pixeles en un monitor, el niño que recibe esa imagen que le habla al igual que otro ser humano reacciona y asimila pensando que es una persona no una representación de la persona. Hasta yo misma he llegado a aceptar un tipo de relación social basado en la interactuación vía skype o cualquier sistema de comunicación virtual que permite la escucha y la visión. Esta claro que el ser humano va a llegar más lejos y está preparando nuevos sistemas que implementen más sentidos en estas relaciones virtuales como el olor o el tacto. Desde el momento en el que somos capaces de actuar mediante un control remoto sobre el entorno, también somos capaces de actuar y hacer sentir a las personas desde otros espacios. Hemos normalizado este tipo de experiencias y aceptado que es parte de la evolución del ser humano. Pero no tenemos que olvidar con son los humano los que crean y dan sentido a la tecnología no podemos decidir solo en base a los datos, los datos y las máquinas no deciden, deciden las personas.

Pero hay cosas que jamas podremos automatizar del todo, como el pensamiento humano, y es que contestar a la pregunta ¿cuándo sabemos si un circulo está recto o curvo?, no está al alcance de la lógica. En películas sobre inteligencia artificial se tratan situaciones futuristas y de ficción en las que el ser humano es capaz de establecer una relación de pareja con un sistema operativo que aprende por si solo y que hasta parece pensar, como en la película "Her" escrita y dirigida por Spike Jonze. Encontramos películas como esta "visionarias" de una situación no muy lejana, en ellas se pone en evidencia el error a parte del avance, son en parte critico de la ciencia ficción que proponen nuevas vías de reflexión. Al ritmo frenético que vamos se podría hablar hasta de suprimir las relaciones sexuales reales por interacciones virtuales entre humanos y máquinas o humanos y humanos, telematicamente. Esto solo puede generar miedo a las relaciones y odio al prójimo a favor de lo que está lejos o lo que no se encuentra.

Esta articulo indaga en la creación de nuevos sistemas de interacción multimedia dentro de las obras de videoarte que conecten la experiencia real con la virtual en un mismo plano, haciendo que el espectador tome una parte activa independientemente de su voluntad de participación asegurándonos así un completo funcionamiento de la obra. Se generan obras en las que el espectador y su presencia en el espacio juegan un papel muy importante en la obra, modificándola y completándola, siendo una experiencia única recibida por el usuario.

Se trata de implicar al espectador en la obra de arte dentro de su plano virtual para generar un espacio de integración entre dos mundos, los cuales generan una experiencia real.

1 Viktor Mayer Schönberger \& Kenneth Cukier (2003). Big Data: La revolución de los datos masivos. Turner, Madrid.

2 Oteiza, J. (1975). Quosque tandem...!, Ensayo de interpretación estética del alma vasca, (3ª Ed.), San Sebastián, Txertoa. 
Obras como las de Hanna Haaslathi y su pieza "Space of two categories" ${ }^{3}$ entre otras representan la idea de interacción que se quiere llevar a cabo y hablan sobre la importancia del cuerpo humano sobre lo tecnológico que se manifiestan a través de la tecnología que hace de puente para entender el cambio de pensamiento que es necesario para la creación actual y el empleo del cuerpo. Nos encontramos actualmente con un cuerpo territorial emancipado en pro de un cuerpo tecnológico. El accidente ocurre cuando perdemos la noción y las posibilidades de nuestro antiguo cuerpo, el cual intenta revalorizar Hanna. El cuerpo juega un papel muy importante en la percepción y tras este genero de obra se refleja gracias al efecto que tiene el propio cuerpo sobre la obra, la cual se muestra reactiva a la figura presente. La captación del espectador en la pieza es inminente, tarda muy poco en reconocer que es su propia figura la que aparece en la pared, con ello se logra la implicación del espectador en la obra involuntariamente e independientemente de que el usuario quiera continuar con el juego la obra ya está completa.

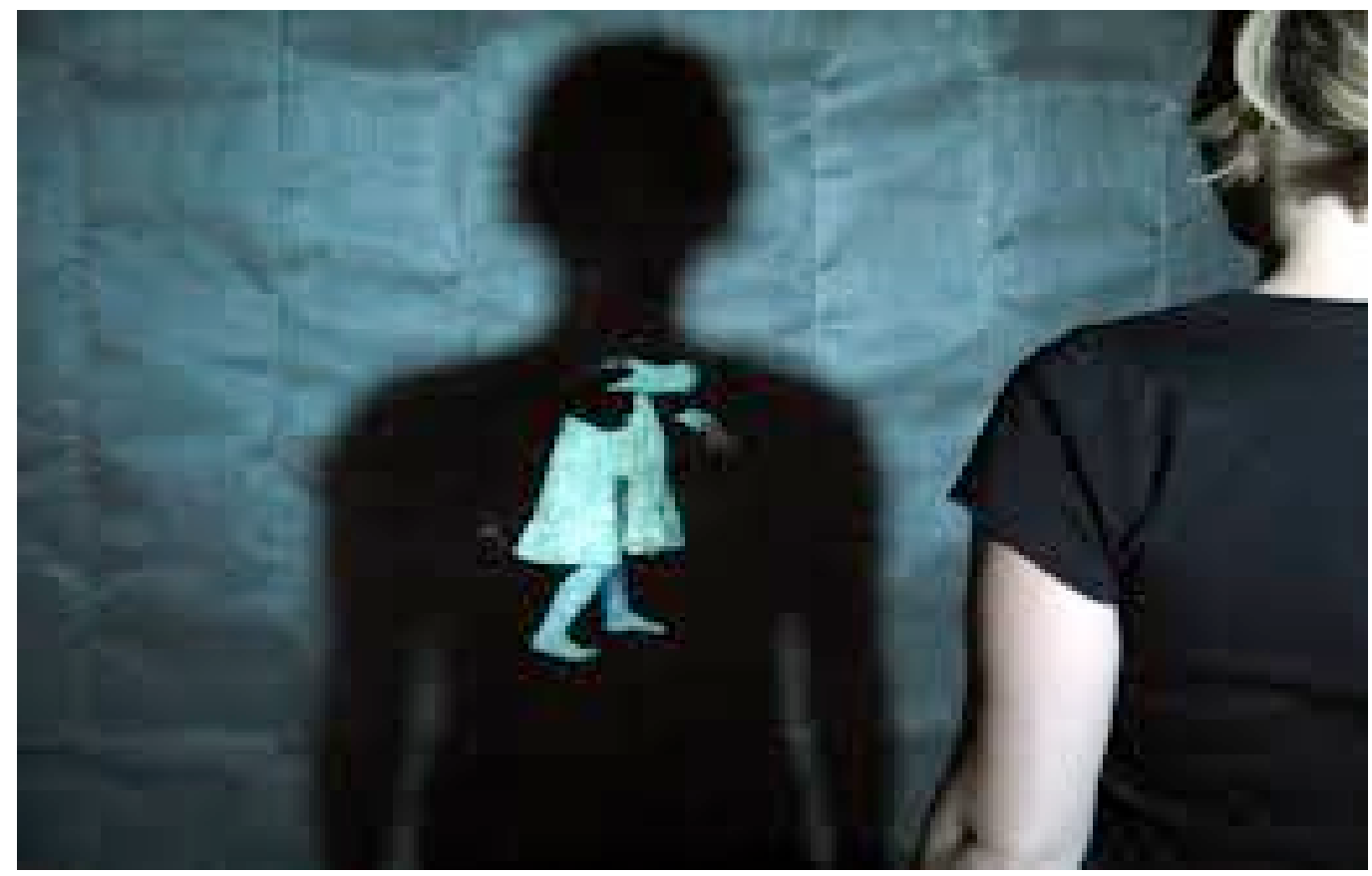

Ilustración 1: Instalación de Hanna "Space of to categories"

Aquí y ahora se quiere avanzar un poco más haciendo que la percepción de la obra sea una experiencia colectiva o cambiante en el tiempo, en una sociedad en la que todo sucede muy rápido, en la que nos valemos de numerosos instrumentos con aplicaciones para ver y percibir el presente, somos incapaces de ver una visión en conjunto.

La obra de la artista Lynn Hershman también puede ser considerada como visionaria, en ella se cuestionan los roles de las personas, la subjetividad sexuada transmitiéndonos la idea de identidades ficticias, una de sus obras más importantes es "Roberta" un personaje ficticio que desarrolló durante 10 años. Lynn es una pionera del arte multimedia e interactivo, y aborda temas como la compenetración del ser humano y las máquinas. A pesar de sus innovadoras aportaciones, la obra de Hershman no ha sido ampliamente reconocida hasta hace poco, con diversos premios y becas de reconocido prestigio, entre los cuales el |DDAA| DAM Digital Art Award ${ }^{4}$, otorgado en 2010 en reconocimiento a la carrera de la artista y sus aportaciones al desarrollo del media art.

3 Haaslathi, H. (2006). Space of two categories [ Instalación multimedia interactiva] Finlandia. Enlace al video: https://vimeo.com/80375243

4 |DDAA| DAM Digital Art Award <http://www.ddaa-online.org/> 


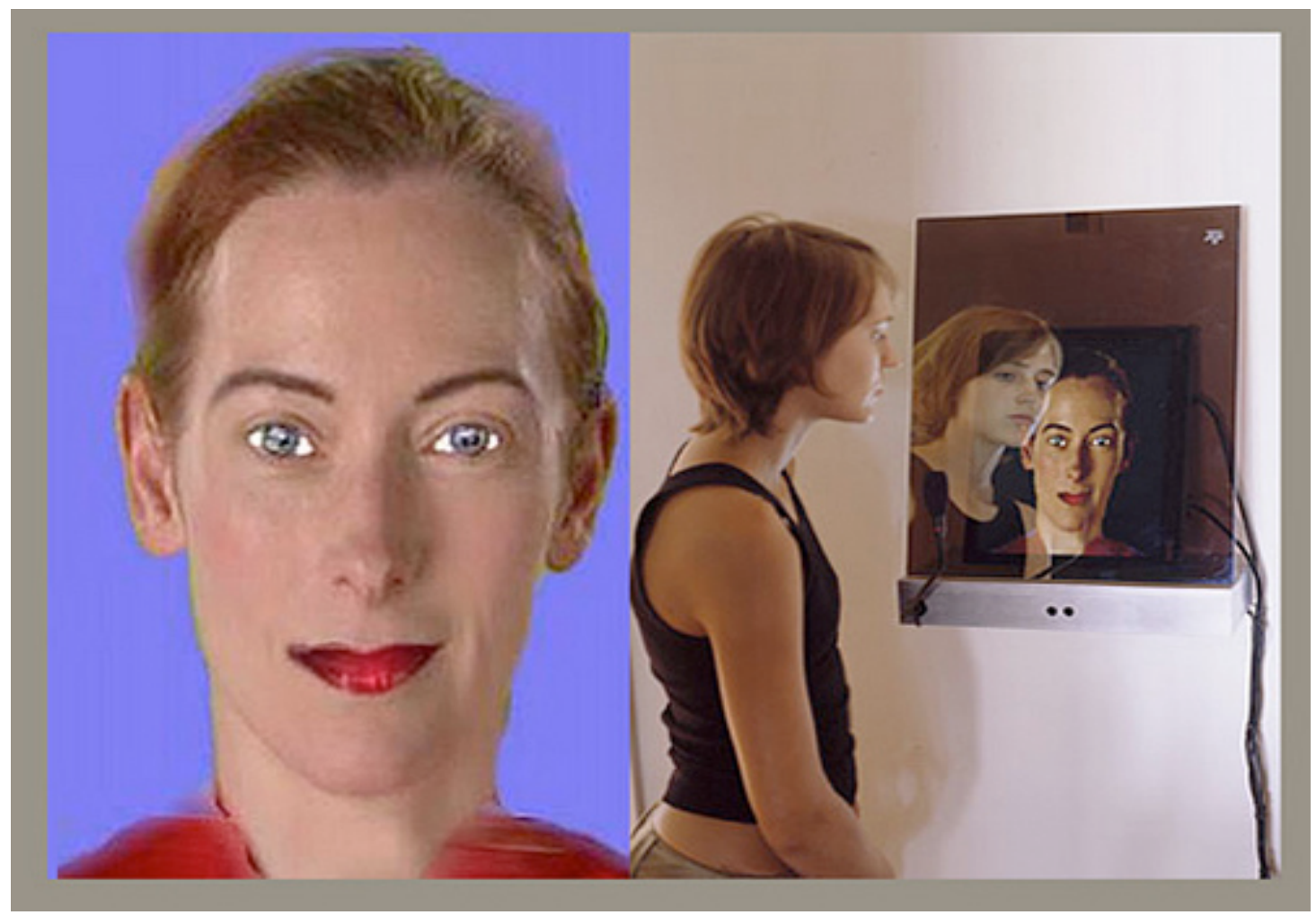

Ilustración 2: Imagen de Dina, el bot de Lynn Hershman, 2004.

En el 2004 creó un bot en red, de inteligencia artificial que se postuló para la presidencia en contra de Bush y Kerry. Nombrado Dina (un juego de palabras con el ADN y las siglas de redes digitales), el lema de la campaña para la DINA era "La inteligencia artificial es mejor que ninguna inteligencia." Dina es expuesto en los museos y los usuarios pueden interactuar con ella.

Existe una ambigüedad de lo virtual con respecto a lo real, cómo es posible que algo intangible y perteneciente a lo virtual genere cosas y experiencias reales, pero los pensamientos también son intangibles y forman parte de lo real. El hándicap de las relaciones virtuales radica en el contacto físico y en la identificación y la relación causa efecto de nuestros actos, tocar, mover no tiene respuesta directa, nuestra presencia no altera lo virtual, por ello vivimos estas experiencias desde el escepticismo y la especulación, desde la simulación de la realidad. Esto cambiará el día que todo este conectado a internet y que cualquier objeto se haya convertido en un cuerpo tecnológico, entonces nos habremos alejado demasiado del ser propio y las relaciones pueden llegar a olvidar como son las relaciones entre seres territoriales, "gracias a la tecnología perdemos el cuerpo propio en beneficio del cuerpo espectral y el mundo propio en beneficio de un mundo virtual" ${ }^{\prime \prime}$. El desarrollo tecnológico ha creado ya el traje de datos "datasuit" y el guante teletacto pero aun no están en nuestras vidas como tal. La obra de Merleau Ponty reflexiona sobre el uso del cuerpo, y elimina lo objetivo y lo subjetivo para vivir la experiencia independientemente de que sea nuestro propio cuerpo o uno prestado. Merleau Ponty elimina lo subjetivo y lo objetivo, se refiere a vivir la experiencia, ya sea en nuestro propio cuerpo o en el del otro ${ }^{6}$.

Cuanto más nos acerquemos a este objetivo de actuar sobre lo real mediante lo virtual más cerca estaremos de vivir experiencias completas en espacios que se encuentran entre dos mundos, espacios que son los que habitamos constantemente, pues nuestras

5 Virilio, P. (1997). El cibermundo, la política de lo peor. Ediciones Cátedra, Madrid. p 51.

6 Ponty, M. (1993). Fenomenología de la percepción. (1ª Ed.). Planeta de Agostini, S.A. Barcelona. 
extensiones corporales forman parte del mundo virtual pero todavía están ancladas a nuestros dedos que son los que interaccionan con este otro espectro. En estos momentos no somos capaces de identificar nuestras acciones como reales, no nos tomamos en serio la realidad, ni la información televisiva, pues habitamos y experimentamos este mundo mitad ficción y mitad realidad, mitad real mitad virtual, lo cual puede generar un vacío existencial, es por eso que buscamos ir más lejos, andar más rápido, estar en varios lugares, actuar sobre cosas lejanas... nuestros dedos están cargados de una energía especial pues conectan dos puertas, pero puede llegar el momento en el que sea todo nuestro cuerpo el que interaccione. La tecnología de captación de movimiento a nivel de usuario, kinect, ya nos ofrece una posibilidad, y se desarrollan sistemas mediante sensores para registrar todos los movimientos de una persona como los empleados por los realizadores de videojuegos. A través de estas obras de arte interactivo se pretende encontrar una unión entre mundos y una formalización de experiencias reales en lo virtual.

\section{OBSOLESCENCIA}

Un buen amigo me dijo en una ocasión:

"que pereza seguir hablando de lo virtual cuando ya está desfasado como diferencia, como difiriendo, como hiperrepresentación en la era hipervisual e hiperdata... ya está 'enunciado' como la imagen inmaterial que es y su construcción de lo Real en oposición a la realidad óntica e indecidible ${ }^{7}{ }^{\prime \prime}{ }^{8}$

Ahora si llevamos estas reflexiones a lo que actualmente se enfrenta nuestro cuerpo nos encontraremos con una serie de cambios o confusiones. El 80 por ciento de la producción microelectrónica se compone de captores, de sensores o de tele-detectores, que nos permiten escuchar a distancia la radio y el micrófono, ver a distancia a través de la cámara y la televisión, tocar a distancia por una parte con el guante teletacto y por otra, con el traje de datos datasuit que permite sentir el cuerpo del otro. Finalmente esta tecnociencia nos encierra en el mundo y nos aísla.

La promesa central del progreso y las mejoras tecnológicas es la del bienestar general de los seres humanos. Es obvio que avances como el de la luz eléctrica, el teléfono o el avión son indiscutiblemente beneficiosos, pero cuando hablamos de el cibermundo y nos topamos con la velocidad de la luz, los fenómenos y los acontecimientos contiguos y derivados son mucho menos perceptibles. Aquí las funciones corporales son paulatinamente 'virtualizadas' mediante los trasplantes de órganos y la clonación, el encuentro a distancia a través de las tele-conferencias, el "teletacto", el "teletraje", estamos de camino al tele-cuerpo produciéndose una pérdida definitiva: la del cuerpo presente.

En este punto comprendemos que somos objeto de una modernización residual que modifica sin contemplaciones el entorno urbano y la naturaleza, a una velocidad en la que no es posible asimilar los cambios de forma lógica y ordenada, pero no es solo eso lo que nos acontece sino que somos objeto también de promesas sobre seres prototipados, universos paralelos y una serie de maravillas que nos distan y nublan nuestra visión real del mundo, es decir nos alejan de nuestro ser más natural. Ante este panorama cabe preguntarse: ¿es que todo va a terminar en el clon, el basurero nuclear y la soledad absoluta del individuo? ¿es que la velocidad del desarrollo tecnológico conduce a una pura fatalidad?

Paul Virilio es muy critico y negativo al respecto, no por que no crea en los beneficios de los avances tecnológicos sino porque ve necesaria la critica del sistema para mantener los pies en la tierra. Desarrolla una teoría de resistencia que se enfoca en la recuperación del otro en la relación de pareja dialogante: "si nuestras sociedades siguen encaminándose hacia (...) la pareja separada y monoparental, no habrá resistencia posible" ${ }^{\prime 9}$. También hace hincapié en la recuperación del mundo natural y a escala humana en contra de los viajes interplanetarios. Y sobre todo enfoca la recuperación por medio de la palabra hablada y escrita. "La salvación vendrá por la escritura y el lenguaje. Si refundamos la lengua, podremos resistir" ${ }^{10}$.

\section{FUENTES REFERENCIALES}

7 Jaques Derrida identifica el termino indecidible o "cadena de los indecidibles", como “...unidades de simulacro, 'falsas' propiedades verbales, nominales o semánticas, que ya no se dejan comprender en la oposición filosófica (binaria) y que no obstante la habitan, la resisten, la desorganizan, pero sin constituir nunca un tercer término, sin dar lugar nunca a una 'solución' en la forma de la dialéctica especulativa"

8 Cabo, Iñigo. Entrevista personal, 25 de Abril 2015.

9 Virilio, P, p. 86.

10 Virilio, P. p. 86. 

II Congreso Anual de Investigadores en Artes Visuales 2015 http://dx.doi.org/10.4995/ANIAV.2015.1061

Oteiza, J. (1975). Quosque tandem...!, Ensayo de interpretación estética del alma vasca, (3ạ Ed.), San Sebastián, Txertoa

Ponty, M. (1993). Fenomenología de la percepción. (1a Ed..). Planeta de Agostini, S.A. Barcelona.

Schönberger, Viktor Mayer \& Cukier, Kenneth (2003). Big Data: La revolución de los datos masivos. Turner, Madrid.

Virilio, P. (1997). El cibermundo, la política de lo peor. Ediciones Cátedra, Madrid. 